\title{
Effects of dielectric permittivities on skin heating due to millimeter
} wave exposure

\author{
Akio Kanezaki ${ }^{1,2}$, Akimasa Hirata*2,3, Soichi Watanabe ${ }^{2}$ and Hiroshi Shirai ${ }^{1}$
}

Address: ${ }^{1}$ Chuo University, Department of Electrical, Electronic, and Communication Engineering, Tokyo, Japan, ${ }^{2}$ National Institute of Information and Communications Technology, EMC group, Tokyo, Japan and ${ }^{3}$ Nagoya Institute of Technology, Nagoya, Department of Computer Science and Engineering, Japan

Email: Akio Kanezaki - akiokanezaki@gmail.com; Akimasa Hirata* - ahirata@nitech.ac.jp; Soichi Watanabe - wata@nict.go.jp; Hiroshi Shirai - shirai@m.ieice.org

* Corresponding author

Published: 23 September 2009

BioMedical Engineering OnLine 2009, 8:20 doi:10.1 186/1475-925X-8-20
Received: 28 April 2009

Accepted: 23 September 2009

This article is available from: http://www.biomedical-engineering-online.com/content/8/I/20

(C) 2009 Kanezaki et al; licensee BioMed Central Ltd.

This is an Open Access article distributed under the terms of the Creative Commons Attribution License (http://creativecommons.org/licenses/by/2.0), which permits unrestricted use, distribution, and reproduction in any medium, provided the original work is properly cited.

\begin{abstract}
Background: Because the possibility of millimeter wave (MMW) exposure has increased, public concern about the health issues due to electromagnetic radiation has also increased. While many studies have been conducted for MMW exposure, the effect of dielectric permittivities on skin heating in multilayer/heterogeneous humanbody models have not been adequately investigated. This is partly due to the fact that a detailed investigation of skin heating in a multilayer model by computational methods is difficult since many parameters are involved. In the present study, therefore, theoretical analyses were conducted to investigate the relationship between dielectric permittivities and MMW-induced skin heating in a one-dimensional three-layer model (skin, fat, and muscle).
\end{abstract}

Methods: Approximate expressions were derived for the temperature elevation and temperature difference in the skin due to MMW exposure from analytical solutions for the temperature distribution. First, the power absorption distribution was approximated from the analytical solution for a one-layer model (skin only). Then, the analytical expression of the temperature in the three-layer model was simplified on the basis of the proposal in our previous study. By examining the approximate expressions, the dominant term influencing skin heating was clarified to identify the effects of the dielectric permittivities. Finally, the effects of dielectric permittivities were clarified by applying partial differentiation to the derived dominant term.

Results: Skin heating can be characterized by the parameters associated with the dielectric permittivities, independently of morphological and thermal parameters. With the derived expressions, it was first clarified that skin heating correlates with the total power absorbed in the skin rather than the specific absorption rate (SAR) at the skin surface or the incident power density. Using Debye-type expression we next investigated the effect of frequency dispersion on the complex relative permittivity of tissue. The parametric study on the total power absorbed in the skin showed that skin heating increases as the static permittivity and static conductivity decrease. In addition, the maximum temperature elevation on the body surface was approximately 1.6 times that of the minimum case. This difference is smaller than the difference caused by the thermal and morphological parameters reported in our previous study.

Conclusion: This paper analytically clarified the effects of dielectric permittivities on the thermally steady state temperature elevation and the temperature difference in the skin of a one-dimensional three-layer model due to MMW exposure. 


\section{Background}

Millimeter waves (MMW: 30-300 GHz) have become one of the attractive communication tools for short-range and high-capacity transmission due to the manufacturing technology advancement of electronic devices. As MMW usage expands, public concern over human exposure rises, and so it has become important to evaluate human safety regarding MMWs. Most MMW power is absorbed near the surface of the human body, leading to a localized temperature elevation near the surface. There have been a few studies on the relationship between the warm sensation and temperature elevation due to MMW exposure. Three general hypotheses of an adequate stimulus for the warm sensation exist, as summarized by Riu et al. [1]: (1) to reach the threshold, the stimulus must produce a fixed temperature elevation in the vicinity of receptors located $150-200 \mu \mathrm{m}$ below the skin surface [2], (2) a stimulus reaches the threshold when it establishes a fixed temperature difference between two layers located at a depth of approximately 200 and 1,000 $\mu \mathrm{m}$ [3], and (3) a stimulus produces a threshold sensation when the temperature elevation at the receptor layer reaches a level that varies with the concurrent level of adaptation [4]. In addition, the rate of temperature elevation could be another factor. However, the determinative factor of warm sensations is unclear. Therefore, a detailed investigation of the temperature elevation and temperature difference near the body surface is required.

Temperature elevation in human tissues due to electromagnetic wave exposure may be estimated from a bioheat equation (BHE) $[5,6]$. On the basis of this equation, the mechanism of warm sensation due to microwave exposure has been discussed by Riu et al. [1] and Foster et al. [7]. They indicated that the body surface temperature elevation in a one-layer model increases with the frequency of the electromagnetic wave. In the MMW region, computational and experimental work has been conducted by Alekseev et al. [8-11]. In [8], they proposed a temperature estimation method by solving a conventional BHE as well as a hybrid bioheat equation (HBHE). HBHE combines the conventional BHE and a scalar effective thermal conductivity equation. Comparing the MMW-induced temperature elevation in the skin with the measured one, the effectiveness of HBHE was shown for low and high perfusion rates. In [11], the temperature elevation in a onedimensional four-layer model was investigated with HBHE.

In our previous study [12], the effect of thermal parameters on the temperature elevation in a three-layer model (skin, fat, muscle) due to MMW exposure was investigated by deriving the analytical solution and its approximation. The main findings in that study were as follows: (1) thermal analysis for a multilayer structure is required even in the MMW region because the temperature elevations for the three-layer model are 1.3-2.8 times larger than those for a one-layer (skin) model and (2) the temperature elevation on the body surface decreases monotonically with the increase of the heat transfer coefficient, terms associated with the blood perfusion, and thermal conductivity.

There have been a few studies on the dielectric permittivities in the MMW region. The variation of the dielectric permittivities of biological tissues $[9,13,14]$ can reach $35 \%$ in the MMW region. However, the effect of the dielectric permittivities on tissue heating in the multilayer model has not been adequately investigated. Moreover, the effect cannot be properly evaluated by our previous approximation [12] because those expressions were derived for the temperature elevation at the body surface only, and the parameters associated with the dielectric permittivities are not independent of the thermal and morphological parameters". For these reasons, additional investigations are required to characterize the dependence of MMWinduced skin heating on the dielectric permittivities.

In the present study, theoretical analyses were conducted for the thermal steady state temperature elevation in order to investigate the relationship between the dielectric permittivities and skin heating in a three-layer model. Continuous MMW was assumed to be incident normal to the body surface as a typical worst-case scenario.

\section{Methods \\ Analytical model and approximation for body surface temperature elevation}

The model, fundamental solution and approximation methods used in our previous study [12] are also used in the present study. A one-dimensional analytical human body model that consists of skin $(n=1)$, fat $(n=2)$, and muscle $(n=3)$ is shown in Fig. 1. A continuous plane wave is incident normal to the skin layer. The temperature distribution can be derived using the $\mathrm{BHE}$, which is expressed for the one-dimensional model as:

$$
\begin{aligned}
& \frac{\partial}{\partial z}\left(\kappa_{n} \frac{\partial T_{n}(z)}{\partial z}\right)+\sigma_{n}|E(z)|^{2}-b_{n}\left\{T_{n}(z)-T_{b}\right\} \\
& +A_{n}=0
\end{aligned}
$$

where $E(\mathrm{z})$ is the root-mean-square electric field $[\mathrm{V} / \mathrm{m}], \sigma_{n}$ is the electric conductivity $[\mathrm{S} / \mathrm{m}], \kappa_{n}$ is the thermal conductivity $\left[\mathrm{W} / \mathrm{m}{ }^{\circ} \mathrm{C}\right], b_{n}$ is the term associated with blood perfusion $\left[\mathrm{W} / \mathrm{m}^{3}{ }^{\circ} \mathrm{C}\right], A_{n}$ is the metabolic heat production $\left[\mathrm{W} / \mathrm{m}^{3}\right]$ of the $n$th layer, and $T_{b}$ is the blood temperature $\left[{ }^{\circ} \mathrm{C}\right]$. Equation (1) can be solved analytically using the Laplace Transform with respect to the $z$ coordinate. $T_{n}(z)$, 


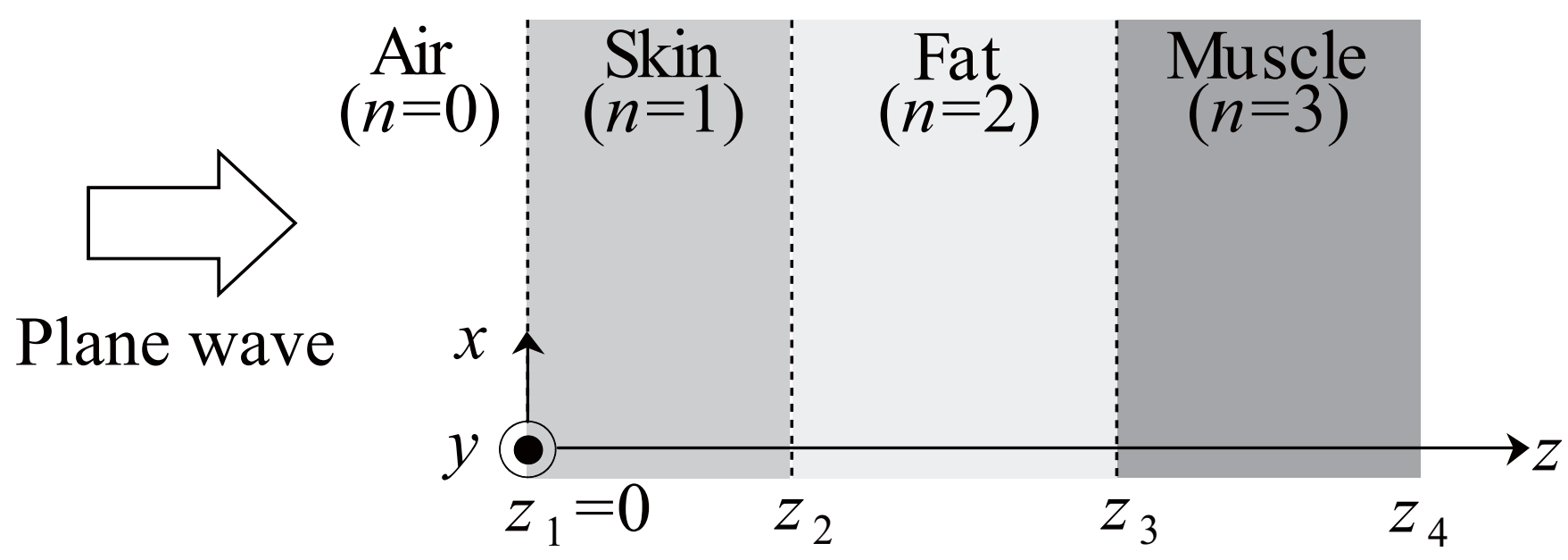

Figure I

Thermal constants and tissue thicknesses in the analytical model ("max"-"typical"-"min"). This model consists of skin $(n=1)$, fat $(n=2)$, and muscle $(n=3)$. The thicknesses of each layer are listed in Table I.

which is the theoretical solution of the temperature distribution in the tissue layer $n$, is derived as follows [12]:

$$
\begin{aligned}
T_{n}(z)= & T_{n}\left(z_{n}\right) \cdot \cosh \sqrt{\frac{b_{n}}{\kappa_{n}}}\left(z-z_{n}\right) \\
& +T_{n}^{\prime}\left(z_{n}\right) \cdot \frac{1}{\sqrt{b_{n} / \kappa_{n}}} \cdot \sinh \sqrt{\frac{b_{n}}{\kappa_{n}}}\left(z-z_{n}\right) \\
& +\left(T_{b}+\frac{A_{n}}{b_{n}}\right) \cdot\left(1-\cosh \sqrt{\frac{b_{n}}{\kappa_{n}}}\left(z-z_{n}\right)\right) \\
& +S_{n}\left(z-z_{n}\right),
\end{aligned}
$$

where

$$
T_{n}^{\prime}\left(z_{n}\right)=\left.\frac{\partial T_{n}\left(z_{n}\right)}{\partial z}\right|_{z=z_{n}}
$$

and $S_{n}\left(z-z_{n}\right)$ is the term associated with the MMW power absorption given by the following equation:

$$
\begin{aligned}
& S_{n}\left(z-z_{n}\right) \\
& =\frac{Z_{0} p \sigma_{n}\left|T_{E n}\right|^{2}}{4 \alpha_{n}^{2} \kappa_{n}-b_{n}}\left\{\frac{-2 \alpha_{\mathrm{n}}}{\sqrt{b_{n} / \kappa_{n}}} \sinh \sqrt{\frac{b_{n}}{\kappa_{n}}}\left(z-z_{n}\right)\right. \\
& \left.+\cosh \sqrt{\frac{b_{n}}{\kappa_{n}}}\left(z-z_{n}\right)-\mathrm{e}^{-2 \alpha_{\mathrm{n}}\left(z-z_{n}\right)}\right\} \\
& +\frac{Z_{0} p \sigma_{n}\left|R_{E n}\right|^{2}}{4 \alpha_{n}^{2} \kappa_{n}-b_{n}}\left\{\frac{2 \alpha_{n}}{\sqrt{b_{n} / \kappa_{n}}} \sinh \sqrt{\frac{b_{n}}{\kappa_{n}}}\left(z-z_{n}\right)\right. \\
& \left.+\cosh \sqrt{\frac{b_{n}}{\kappa_{n}}}\left(z-z_{n}\right)-\mathrm{e}^{2 \alpha_{\mathrm{n}}\left(z-z_{n}\right)}\right\} \\
& +\frac{z_{0} p \sigma_{n} X_{1}}{4 \beta_{n}^{2} \kappa_{n}+b_{n}} \\
& \left\{-\mathrm{e}^{-\sqrt{\frac{b_{n}}{\kappa_{n}}}\left(z-z_{n}\right)}-\mathrm{e}^{\sqrt{\frac{b_{n}}{\kappa_{n}}}\left(z-z_{n}\right)}+2 \cos 2 \beta\left(z-z_{n}\right)\right\} \\
& +\frac{2 Z_{0} p \sigma_{n} \beta_{n} X_{2}}{4 \beta_{n}^{2} \kappa_{n}+b_{n}}\left\{-\sqrt{\frac{b_{n}}{\kappa_{n}}} e^{-\sqrt{\frac{b_{n}}{\kappa_{n}}}\left(z-z_{n}\right)}\right. \\
& \left.-\sqrt{\frac{b_{n}}{\kappa_{n}}} e^{\sqrt{\frac{b_{n}}{\kappa_{n}}}\left(z-z_{n}\right)}+\frac{\sin 2 \beta_{n}\left(z-z_{n}\right)}{\beta_{n}}\right\}
\end{aligned}
$$

where 


$$
\begin{aligned}
& X_{1}=\operatorname{Re}\left(T_{E n}\right) \operatorname{Re}\left(R_{E n}\right)+\operatorname{Im}\left(T_{E n}\right) \operatorname{Im}\left(R_{E n}\right), \\
& X_{2}=\operatorname{Im}\left(T_{E n}\right) \operatorname{Re}\left(R_{E n}\right)-\operatorname{Re}\left(T_{E n}\right) \operatorname{Im}\left(R_{E n}\right) .
\end{aligned}
$$

Note that $Z_{0}$ is the free-space wave impedance $[\Omega], p$ is the incident power density $\left[\mathrm{W} / \mathrm{m}^{2}\right], \alpha_{n}$ is the attenuation constant of the $n$-th layer $\left[\mathrm{m}^{-1}\right], \beta_{n}$ is the phase constant of the $n$-th layer $[\mathrm{rad} / \mathrm{m}], T_{E n}$ is the transmission coefficient from layer $n$ - 1 to $n$, and $R_{E n}$ is the reflection coefficient from layer $n+1$ to $n$.

Although analytically exact, solution (2) is difficult to apply to a parametric study. For this reason, a simpler analytical solution is desirable. Since the MMW power absorption is confined within the skin surface, the absorbed power can be derived from a simpler analytical solution for the one-layer (skin only) model. This physical observation can be used for an approximate solution [12], and so the body surface temperature elevation $\Delta T_{3 L}$ due to MMW exposure in the three-layer model can be written as follows:

$$
\begin{aligned}
& \Delta T_{3 L}=\frac{Z_{0} p \sigma_{1}\left|T_{E 10}\right|^{2}}{4 \alpha_{1}^{2}} \cdot \frac{2 \alpha_{1}(A+B \cdot D)-C \cdot D}{A \cdot E+D} \\
& \left\{\begin{array}{l}
A=\frac{z_{3}-z_{2}}{\kappa_{2}}\left(\frac{b_{2}\left(z_{3}-z_{2}\right)}{2 \sqrt{b_{3} \kappa_{3}}}+1\right)+\frac{1}{\sqrt{b_{3} \kappa_{3}}} \\
B=\frac{z_{2}}{\kappa_{1}}, C=\frac{1}{\kappa_{1}} \\
D=b_{2}\left(z_{3}-z_{2}\right)\left(\frac{z_{3}-z_{2}}{2 \kappa_{2}}+\frac{1}{\sqrt{b_{3} \kappa_{3}}}\right)+1 \\
E=h+b_{1} z_{2},
\end{array}\right.
\end{aligned}
$$

where the transmission power at the air-skin interface is given by

$$
\begin{aligned}
\left|T_{E 10}\right|^{2} & =\left|\frac{2 Z_{1}}{Z_{0}+Z_{1}}\right|^{2} \\
& =\frac{4}{\left|\varepsilon_{r 1}^{*}\right|+\sqrt{2\left(\left|\varepsilon_{r 1}^{*}\right|+\varepsilon_{r 1}\right)}+1}
\end{aligned}
$$

with

$$
\varepsilon_{r 1}^{*}=\varepsilon_{r 1}-j \frac{\sigma}{\omega \varepsilon_{0}}
$$

and $h$ is the heat transfer coefficient $\left[\mathrm{W} / \mathrm{m}^{2}{ }^{\circ} \mathrm{C}\right], Z_{n}$ is the wave impedance $[\Omega], \varepsilon_{r 1}$ is the relative permittivity in the skin tissue, and $\varepsilon_{0}$ is the permittivity in the vacuum $[\mathrm{F} / \mathrm{m}]$.

\section{Approximate expressions for temperature distributions in the skin tissue}

Temperature distribution $T_{1}\left(z_{\mathrm{s}}\right)_{\mathrm{B}}$ in the skin tissue before exposure can be approximated by the following expression:

$$
T_{1}\left(z_{\mathrm{s}}\right)_{B}=T_{1}(0)_{B}+\left\{T_{1}(0)_{B}-T_{\text {Air }}\right\} \frac{h}{\kappa_{1}} z_{\mathrm{s}^{\prime}}
$$

where $0 \leq z_{\mathrm{s}}<z_{2}$. The subscript $\mathrm{B}$ refers to the condition before exposure. The temperature elevation in the skin tissue $\Delta T\left(z_{\mathrm{s}}\right)$ is approximated as follows:

$$
\begin{aligned}
\Delta T\left(z_{\mathrm{s}}\right) & =\Delta T_{3 L}\left(1+\frac{h}{\kappa_{1}} z_{\mathrm{s}}\right) \\
& +\frac{Z_{0} p \sigma_{1}\left|T_{E 10}\right|^{2}}{2 \alpha_{1} \kappa_{1}} \cdot\left\{\frac{1}{2 \alpha_{1}}-\left(z_{\mathrm{s}}+\frac{e^{-2 \alpha_{1} z_{\mathrm{s}}}}{2 \alpha_{1}}\right)\right\} .
\end{aligned}
$$

Furthermore, the temperature difference, Diff. $T\left(\Delta z_{\mathrm{s}}\right)$, between $z_{\mathrm{s} 1}$ and $z_{\mathrm{s} 2}$ due to the continuous MMW exposure can be approximated as:

$$
\begin{aligned}
\operatorname{Diff} . T\left(\Delta z_{\mathrm{s}}\right) & =T_{1}\left(z_{\mathrm{s} 1}\right)-T_{1}\left(z_{\mathrm{s} 2}\right) \\
& =\left\{T_{\text {Air }}-T_{1}(0)_{B}-\Delta T_{3 L}\right\} \frac{h}{\kappa_{1}} \Delta z_{\mathrm{s}} \\
& +\frac{Z_{0} p \sigma_{1}\left|T_{E 10}\right|^{2}}{2 \alpha_{1} \kappa_{1}} \cdot\left\{\Delta z_{\mathrm{s}}-\frac{e^{-2 \alpha_{1} z_{\mathrm{s} 1}-e^{-2 \alpha_{1} z_{\mathrm{s} 2}}}}{2 \alpha_{1}}\right\},
\end{aligned}
$$

where $T_{1}(0)_{B}$ is the body surface temperature $\left[{ }^{\circ} \mathrm{C}\right]$ before exposure and $T_{A i r}$ is the air temperature $\left[{ }^{\circ} \mathrm{C}\right]$.

It is difficult to investigate the effect of the dielectric permittivities on skin heating (i.e., temperature elevation and temperature difference that may cause the warm sensation, as noted previously) using the above expressions because the terms associated with the dielectric permittivities are not independent. Therefore, further approximate expressions for the temperature distributions must be derived to investigate the effect of the dielectric permittivities on skin heating. The attenuation constant of skin tissue, $\alpha_{1}[1 / \mathrm{m}]$, ranges from 1,000 to 4,000 in the MMW region, and therefore, $1 / \alpha_{1}{ }^{2}$ may be approximately equal to zero in the equations of body surface temperature elevation (7), temperature elevation distribution in the skin tissue (12), and temperature difference in the skin tissue 
(13). These equations can also be approximated by the following expressions:

$$
\begin{aligned}
& \Delta \hat{T}_{3 L}=\frac{Z_{0} p \sigma_{1}\left|T_{E 10}\right|^{2}}{2 \alpha_{1}} \cdot \frac{A+B \cdot D}{A \cdot E+D} \\
& \Delta \hat{T}\left(z_{\mathrm{s}}\right)=\frac{Z_{0} p \sigma_{1}\left|T_{E 10}\right|^{2}}{2 \alpha_{1}} \\
& \left\{\frac{A+B \cdot D}{A \cdot E+D}\left(1+\frac{h}{\kappa_{1}} z_{\mathrm{s}}\right)-\frac{1}{\kappa_{1}}\right\}, \\
& \operatorname{Diff.T}\left(\Delta z_{\mathrm{s}}\right)=\left\{T_{A i r}-T_{1}(0)_{B}\right\} \frac{h}{\kappa_{1}} \Delta z_{\mathrm{s}} \\
& +\frac{Z_{0} p \sigma_{1}\left|T_{E 10}\right|^{2}}{2 \alpha_{1}} \cdot\left\{-\frac{A+B \cdot D}{A \cdot E+D} \frac{h}{\kappa_{1}} \Delta z_{\mathrm{s}}+\frac{\Delta z_{\mathrm{S}}}{\kappa_{1}}\right\} .
\end{aligned}
$$

Thermal, morphological, and electrical parameters The biological parameters for our present calculations were chosen from the literature [15-22], and are listed in Table 1. The rationale for these choices is discussed in [12]. The conditions ("max" and "min"), in which the maximum and minimum body surface temperature elevations are observed, were determined in [12]. In addition, the set of commonly used parameters are named "typical" $[20,21,23]$.

The dielectric permittivities of tissues were determined on the basis of [13]. The complex relative permittivity in the skin tissues "dry," "(dry + wet)/2," and "wet" (see Fig. 2) are applied to the conditions "max," "typical," and "min." In contrast, the variations in the dielectric permittivities in fat and muscle tissues are not considered because the differences in the dielectric permittivities of fat and muscle tissues have little influence on the MMW power absorption [9].

\section{Results and Discussion}

\section{Approximate expressions of skin heating}

The temperature distributions in the skin tissue before exposure using an analytical exact solution (2) and its approximation (11) are shown in Fig. 3. Good agreement is shown between the analytical solution and the approximation, and relative errors are at most $1 \%$. The temperature elevation in the skin tissue due to MMW exposure at (a) $30 \mathrm{GHz}$ and (b) $300 \mathrm{GHz}$ using the analytical exact solution (2) and its approximation (15) are shown in Fig. 4. The incident power density of MMW was chosen as 50 $\mathrm{W} / \mathrm{m}^{2}$. As seen from Figs. 4(a) and (b), the errors attributed to the approximation increase as the frequency decreases. This tendency may be due to the increase in penetration depth of the skin tissue. The relative errors of the approximations are at most $15 \%$ under the worst condition, such as thin skin thickness and lower-frequency $(30 \mathrm{GHz})$ illumination. The validity of our approximation is confirmed, and the temperature distributions before the exposure and the temperature elevations in the skin tissue due to continuous exposure calculated by the approximate expressions (11) and (15) are in satisfactory agreement with those from the analytical solution (2). Therefore, one can utilize the derived approximated formula (15) and (16) for the analytical evaluation of the effect of the dielectric permittivities on skin heating.

Let us further discuss the approximate expressions for the temperature distributions in the skin tissue. As is evident from equations (15) and (16), the term associated with dielectric permittivities $Z_{0} p \sigma_{1}\left|T_{E 10}\right|^{2} / 2 \alpha_{1}$ is independent of the morphological and thermal parameters, and this term is the only factor of skin heating. Assuming that forward propagation is the dominant contribution in the skin layer, as discussed above, the distribution of the specific absorption rate (SAR $[\mathrm{W} / \mathrm{kg}]$ ) in the skin is derived as

$$
\begin{aligned}
\operatorname{SAR}(z) & =\frac{\sigma_{1}|E(z)|^{2}}{\rho_{1}} \\
& \cong \frac{Z_{0} p \sigma_{1}\left|T_{E 10}\right|^{2} \cdot e^{-2 \alpha_{1} z}}{\rho_{n}}
\end{aligned}
$$

Table I: Thermal constants and tissue thicknesses in the analytical model ("max"-"typical"-"min").

\begin{tabular}{lcccc}
\hline & $\boldsymbol{h}$ (surface) & $\boldsymbol{b}_{\boldsymbol{n}}$ & $\kappa_{\mathbf{n}}$ & $\mathbf{z}_{\mathbf{n + 1}-\mathbf{z}_{\mathbf{n}}[\mathbf{m m}]}$ \\
\hline $\operatorname{skin}(n=1)$ & $1-10-15$ & $6800-9100-9100$ & $0.26-0.42-0.50$ & $0.7-1.0-1.3$ \\
fat $(n=2)$ & - & $800-1700-2100$ & $0.20-0.25-0.27$ & $6.3-3.5-0.7$ \\
Muscle $(n=3)$ & - & $1300-2700-3500$ & $0.43-0.50-0.55$ & $53.0-55.5-58.0$ \\
\hline
\end{tabular}

Detailed discussions are described in [13]. The conditions, "max" and "min," in which the maximum and minimum body surface temperature elevations were observed, were determined in [12]. In addition, the set of parameters commonly used are named "typical" [20,21,23]. 


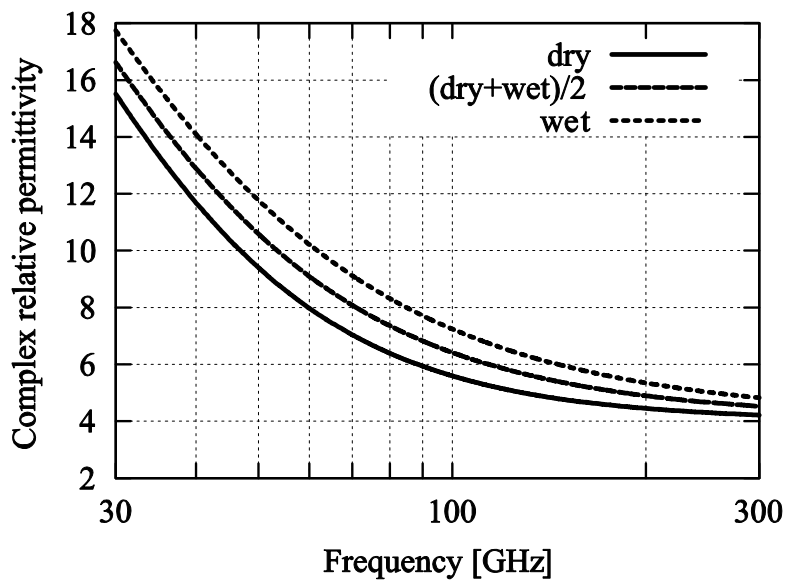

(a)

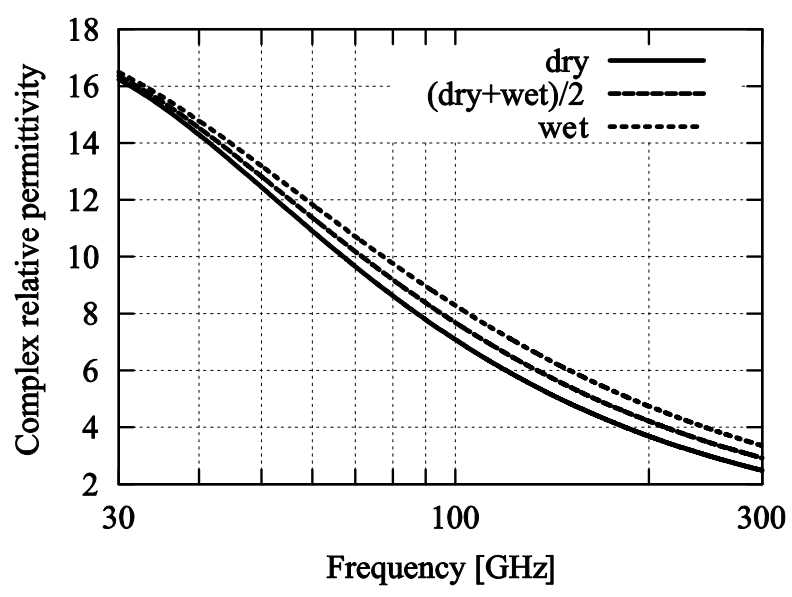

(b)

\section{Figure 2}

Frequency characteristics of the complex relative dielectric permittivity in skin tissue (Gabriel et al. [9]). (a) Real part. (b) Imaginary part.

where $\rho_{1}\left[\mathrm{~kg} / \mathrm{m}^{3}\right]$ is the mass density of the skin tissue. Then, the total absorption power in the skin tissue due to MMW exposure $P_{t}$ is expressed as

$$
\begin{aligned}
P_{t} & =\int_{z_{1}=0}^{z_{2}} \rho(z) \operatorname{SAR}(z) d z \\
& =\frac{Z_{0} p \sigma_{1}\left|T_{E 10}\right|^{2}}{\rho_{n}} \int_{0}^{z_{2}} e^{-2 \alpha_{1} z} d z .
\end{aligned}
$$

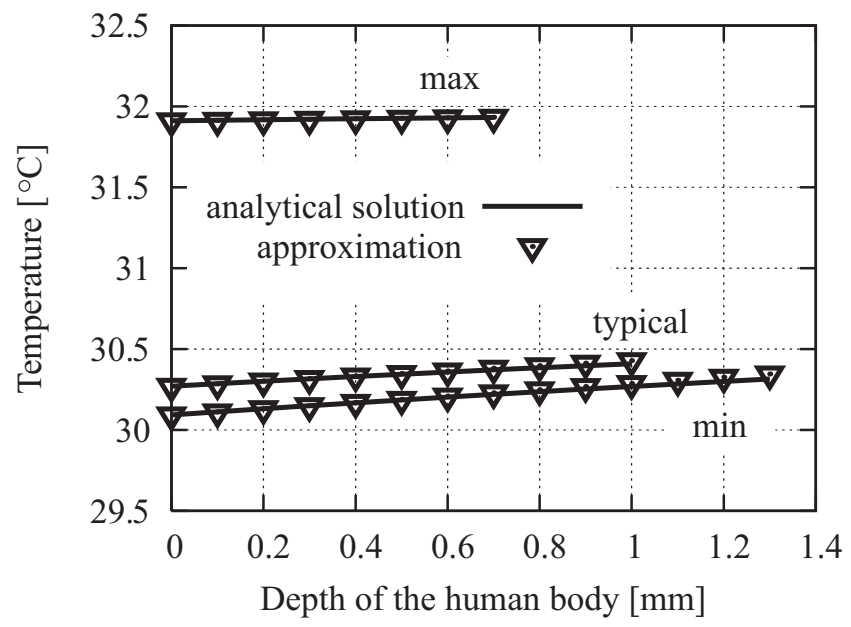

Figure 3

Temperature distributions in the skin tissue before exposure. Comparison between the results obtained from the analytical exact solution (2) and the derived approximate expression (12).

For values of $z_{2}$ shown in Table $1, \alpha_{1} z_{2} \gg \alpha_{1} z_{1}=0$ in the $\mathrm{MMW}$ region. Therefore, the upper integration limit can be ignored to obtain

$$
P_{t} \cong \frac{Z_{0 p \sigma_{1}\left|T_{E 10}\right|^{2}}}{2 \alpha_{1}} .
$$

Consequently, the temperature elevation $\Delta \hat{T}_{1}\left(z_{\mathrm{s}}\right)$ and the temperature difference Diff. $\hat{T}\left(\Delta z_{\mathrm{s}}\right)$ at the location where the warm sensation occurs, correlates only with the total absorption power in the skin. In other words, it is possible to evaluate the effect of the dielectric permittivities on skin heating by the total absorption power in the skin layer per unit area rather than the incident power density or SAR values per unit area at the body surface. Note that the total absorption power per unit area in the skin corresponds to the total energy absorbed per unit area in the skin since we consider the temperature elevation in the thermal steady state.

It is worth comparing our finding in MMW with the corresponding results in microwave frequencies. For frequencies up to $3 \mathrm{GHz}$, the peak $10 \mathrm{~g}$ or mass-averaged SAR is used as a metric in the IEEE standards [24]. One of the rationales for this is the correlation between the 10-g averaged SAR and local temperature elevation. However, such an indicator may be inappropriate for the MMW region, because of the small penetration depth. Our analytical formula shows that the temperature elevation in skin can 


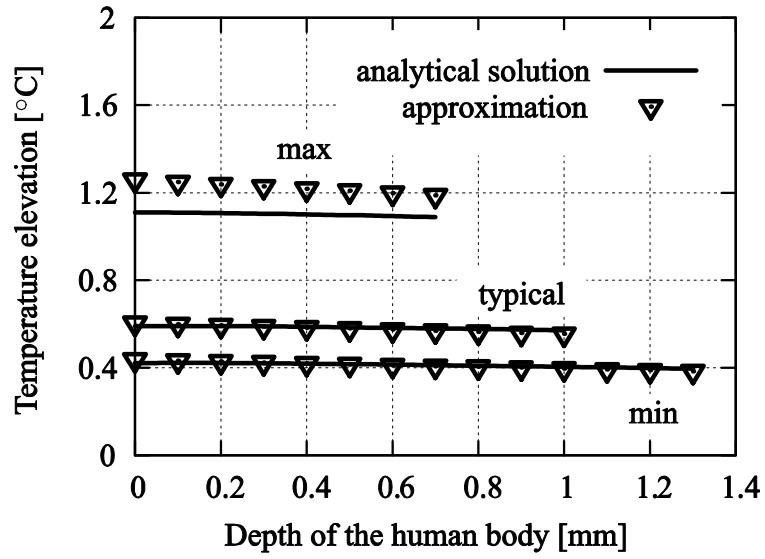

(a)

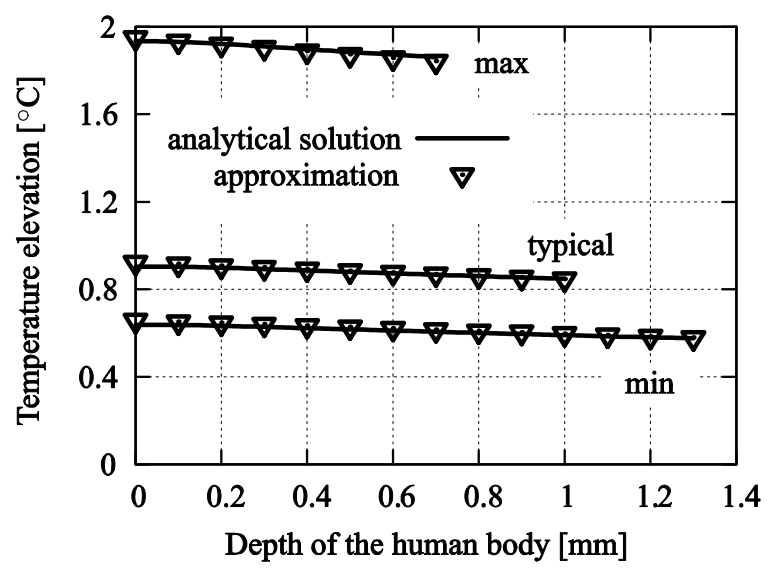

(b)

\section{Figure 4}

Temperature elevations in the skin tissue due to MMW exposure. These are calculated from the analytical exact solution (2) and approximate expression (12) under the conditions of "max", "typical" and "min" in Table I. The incident power density $p$ is $50 \mathrm{~W} / \mathrm{m}^{2}$. (a) $30 \mathrm{GHz}$ (b) 300

$\mathrm{GHz}$

be estimated using the total power absorbed in the skin, instead of the mass-averaged SAR used for microwaves.

\section{Effects of electrical parameters on skin heating}

The total absorption power is determined by the electric conductivity $\sigma_{1}$ in the skin tissue, the attenuation constant $\alpha_{1}$ in the skin tissue, and the transmission coefficient $T_{E 10}$ at the air-skin interface, all of which are related to the dielectric permittivities. Among these parameters, $T_{E 10}$ is the most dominant factor. Therefore, the effects of the dielectric permittivities on skin heating can be investigated by using $T_{E 10}$. Because biological tissues contain a certain amount of water, which exhibits a frequency dispersive nature in the MMW region, the relative dielectric permittivity of biological tissues can be adequately approximated by a Debye-type formula $[10,11]$ :

$$
\begin{aligned}
\varepsilon_{r}^{*} & =\varepsilon_{\infty}+\frac{\varepsilon_{s}-\varepsilon_{\infty}}{1+j \omega \tau}+\frac{\sigma_{s}}{j \omega \varepsilon_{0}} \\
& =\varepsilon_{\infty}+\frac{\varepsilon_{s}-\varepsilon_{\infty}}{1+(\omega \tau)^{2}}-j\left\{\frac{\omega \tau\left(\varepsilon_{s}-\varepsilon_{\infty}\right)}{1+(\omega \tau)^{2}}+\frac{\sigma_{s}}{\omega \varepsilon_{0}}\right\} \\
& =\varepsilon^{\prime}-j \varepsilon^{\prime \prime}
\end{aligned}
$$

where $\omega[\mathrm{rad} / \mathrm{s}]$ is the angular frequency, $\tau[\mathrm{s}]$ is the relaxation time, $\varepsilon_{8}$ is the relative permittivity at $\omega \tau>>1, \varepsilon_{s}\left(>\varepsilon_{8}\right)$ is the static relative permittivity (at $\omega \tau<<1$ ), and $\sigma_{s}[\mathrm{~S} / \mathrm{m}]$ is the static conductivity. The effects of $\varepsilon_{s^{\prime}} \tau$, and $\sigma_{s}$ in equation (20) on skin heating are especially considered. It is clear that the relative permittivities $\varepsilon^{\prime}$ and $\varepsilon$ " increase with $\varepsilon_{\mathrm{s}}$ and $\sigma_{s}$. The partial derivatives of $\varepsilon^{\prime}$ and $\varepsilon^{\prime \prime}$ with respect to $\tau$ are given as follows:

$$
\begin{gathered}
\frac{\partial \varepsilon^{\prime}}{\partial \tau}=-\frac{2 \omega^{2}\left(\varepsilon_{s}-\varepsilon_{\infty}\right) \tau}{\left\{1+(\omega \tau)^{2}\right\}^{2}<0,} \\
\frac{\partial \varepsilon^{\prime \prime}}{\partial \tau}=-\frac{\omega\left(\varepsilon_{s}-\varepsilon_{\infty}\right)\left\{1-(\omega \tau)^{2}\right\}}{\left\{1+(\omega \tau)^{2}\right\}^{2}}<0,
\end{gathered}
$$

since $1<(\omega \tau)^{2}$ for the MMW region [9-11]. Accordingly, $\varepsilon^{\prime}$ and $\varepsilon^{\prime \prime}$ decrease monotonically. As seen in equation (9), the complex relative permittivity $\varepsilon_{r}{ }^{*}$ is included in the denominator of $T_{E 10}$. Thus, skin heating increases as relaxation time $\tau$ increases.

Let us now discuss some parameters of the effect of dielectric permittivities on the temperature elevation. Frequency dispersion characteristics of dielectric permittivities were reported in $[9,13,14]$ and used to calculate the body surface temperature elevations and absorbed powers. These values are shown in Figs. 5 and 6, respectively. Cases exist in which the dielectric permittivities are as much as $35 \%$ larger than the values for the 'dry' skin reported by Gabriel [13]. In contrast, the variations in body surface temperature elevations and absorbed powers were less than $6 \%$ from the results using "Gabriel (dry)." The same conclusion was drawn for the temperature difference in skin tissue for other thermal and morphological parameters, 


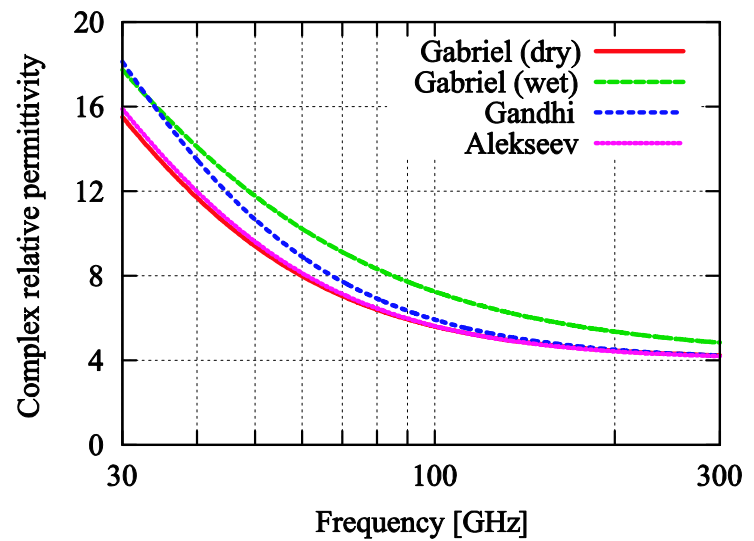

(a)

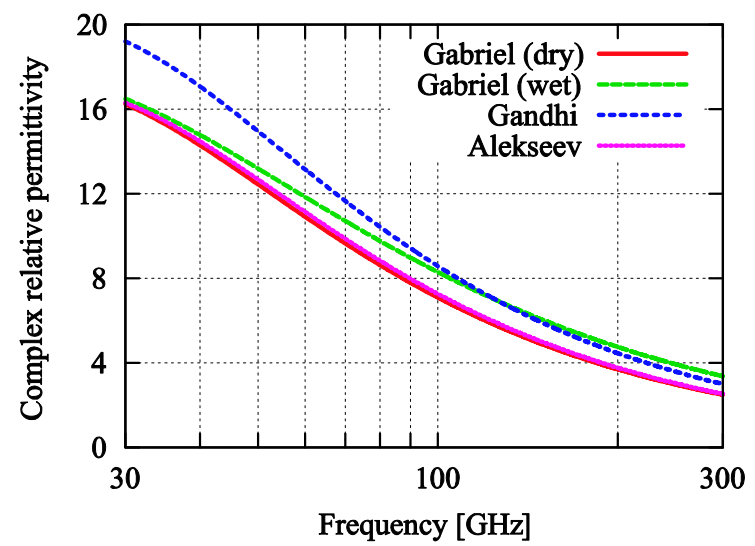

(b)

Figure 5

Frequency characteristics of the dielectric permittivities in skin tissue [9-II]. (a) Real part, (b) Imaginary part of the relative dielectric permittivity.

because these parameters are independent of the dielectric permittivities (see equations (15) and (16)).

Both the temperature elevation $\Delta \hat{T}_{1}\left(z_{s}\right)(15)$ and the temperature difference Diff. $\hat{T}\left(\Delta z_{s}\right)(16)$ are found to increase monotonically with the frequency for the following reasons. The complex relative permittivity in the denominator of $T_{E 10}$ decreases monotonically with the increase in frequency (see Fig. 2). Then, the total absorption power increases with the frequency, which is attributed to the increase in transmission coefficient $\mathrm{TE}_{10}$. From Fig. 6(a), it

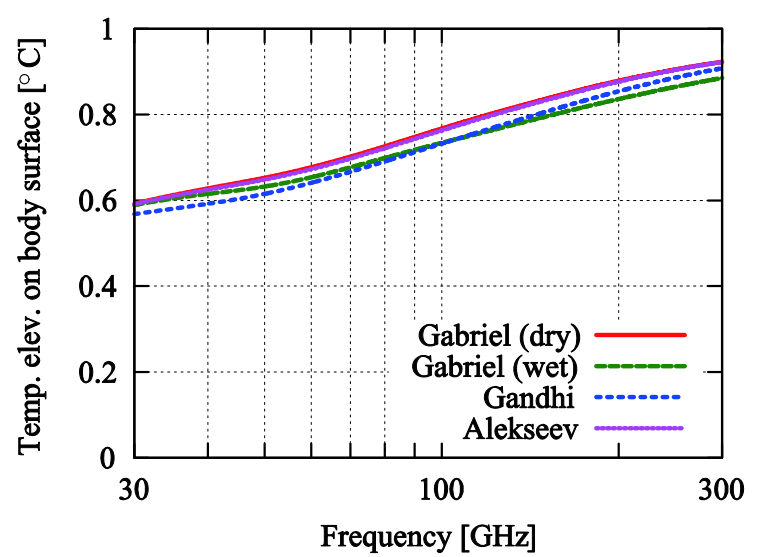

(a)

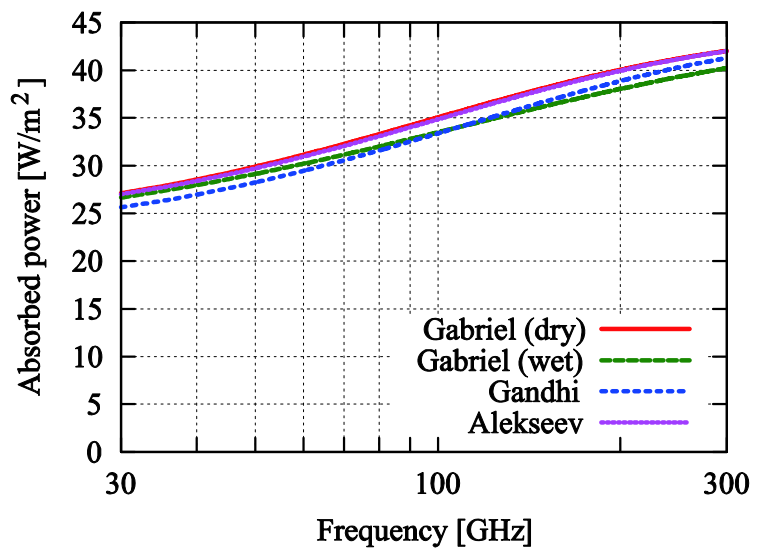

(b)

Figure 6

Frequency characteristics of (a) body surface temperature elevations and (b) total absorption power. These are calculated from the analytical solution (2) and $Z_{0} p \sigma_{\mid}\left|T_{E \mid 0}\right|^{2} / 2 \alpha_{1}$. The power density $p$ is $50 \mathrm{~W} / \mathrm{m}^{2}$, the parameter condition is "typical" in Table I, and the dielectric permittivities in Figure 5 are from [9-II].

is also confirmed that the body surface temperature elevations increase monotonically with the frequency.

The above results show that the maximum temperature elevation on the body surface is approximately 1.6 times the minimum value, although the body surface temperature elevation for thermal constants and tissue thicknesses varies approximately by 3 times [12]. Therefore, the effects of the dielectric permittivities on skin heating are less than those of the thermal constants and tissue thicknesses. 


\section{Conclusion}

This present study investigated the effects of dielectric permittivities on the temperature elevation and temperature difference in the thermal steady state in the skin layer of a one-dimensional three-layer model (skin, fat, muscle) for MMW exposure. Approximate expressions were derived around the location near the warm sensation receptors from the analytical solution for BHE. The derived expressions exhibit clearly that the dielectric permittivity is independent from morphological and thermal parameters. The temperature distributions in the skin tissue by our approximate expressions agree well with those derived from the analytical solution, and the relative errors are at most $15 \%$.

Using our approximate expressions, it is shown that local skin heating can be correlated with total absorption power in the skin per unit area rather than that of the incident power density or that of SAR values per unit area at the body surface. Note that the total absorption power per unit area in the skin corresponds to the total energy absorbed per unit area in the skin since we consider the temperature elevation in the thermal steady state. Therefore, the effects of the dielectric permittivities on skin heating can be evaluated by the total absorption power in the skin tissue only. This measure is similar to the massaveraged SAR used as a metric in the safety guidelines for microwaves [24]. The primary difference is attributed to the penetration depth of electromagnetic waves, resulting in a different SAR averaging region.

Debye-type approximation was introduced to consider the nature of the frequency dispersion of the tissue's dielectric permittivities, and the parametric study was conducted to see the effect of the dielectric permittivities on skin heating. The effects of the dielectric permittivities on skin heating are concluded to be less than those due to the thermal constants and tissue thicknesses.

Future work is to discuss the variability of MMW thermal sensation based on the finding in the present study.

\section{Competing interests}

The authors declare that they have no competing interests.

\section{Authors' contributions}

AK has made contributions to the conception, analysis, and interpretation and has been involved in drafting the manuscript. $\mathrm{AH}$ has been involved in thermal modeling and its interpretation and revising the manuscript for critically important intellectual content. SW and HS have supervised this project and have been involved in revising the manuscript. All authors have read and approved the final manuscript.

\section{Acknowledgements}

This research was partially supported by the Strategic Information and Communication R\&D Promotion Programme (SCOPE), Scientific Research Grant-In-Aid (No. 20360176) from the Japan Society for the Promotion of Science, and 2008 Chuo University Grant for Special Research.

\section{References}

I. Riu PJ, Foster KR, Blick DW, Adair ER: A thermal model for human thresholds of microwave-evoked warmth sensations. Bioelectromagnetics 1997, I 8:578-583.

2. Hendler E, Hardy JD: Infrared and microwave effects on skin heating and temperature sensation. IRE Trans Med Electron 1960, 7:|43-152.

3. Hendler E, Hardy JD, Murgatroyd D: Skin heating and temperature sensation produced by infrared and microwave irradiation. In Temperature: Its Measurement and Control in Science and Industry Edited by: Herzfeld CM. New York: Reinhold; 1963:21 I.

4. Eijkman ED, Vendrik AJH: Dynamic behavior of the warmth sense organ. J Exp Psychol I 96I, 62:403-408.

5. Pennes $\mathrm{HH}$ : Analysis of tissue and arterial blood temperature in resting forearm. J Appl Physiol 1948, I:93-I22.

6. Hoque M, Gandhi OP: Temperature distributions in the human leg for VLF-VHF exposures at the ANSI recommended safety levels. IEEE Trans Biomed Eng 1988, 35:442-449.

7. Foster KR, Lozano-Nieto A, Riu PJ, Appendix by Ely TS: Heating of tissue by microwaves: A model analysis. Bioelectromagnetics 1998, 19:420-428.

8. Alekseeve SI, Radzievsky AA, Szabo I, Ziskin MC: Local heating of human skin by millimeter waves: Effect of blood flow. Bioelectromagnet 2005, 26:489-50I.

9. Alekseev SI, Ziskin MC: Human skin permittivity determined by millimeter wave reflection measurements. Bioelectromagnetics 2007, 28:33I-339.

10. Alekseev SI, Radzievsky AA, Logani MK, Ziskin MC: Millimeter wave dosimetry of human skin. Bioelectromagnetics 2008, 29:65-70.

II. Alekseev SI, Ziskin MC: Influence of blood flow and millimeter wave exposure on skin temperature in different thermal models. Bioelectromagnet 2009, 30:52-58.

12. Kanezaki A, Watanabe S, Hirata A, Shirai H: Theoretical analysis for temperature elevation of human body due to millimeter wave exposure. Proc. Cairo Int'l Biomed Eng Conf 2008:SB-62.

13. Gabriel S, Lau RW, Gabriel C: The dielectric properties of biological tissues: III. Parametric models for the dielectric spectrum of tissues. Phys Med Biol 1996, 41:2271-2293.

14. Gandhi OP, Riazi A: Absorption of millimeter waves by human beings and its biological implications. IEEE Trans Microwave Theory Tech 1986, 34:228-235.

15. Fiala D, Lomas KJ, Stohrer M: A computer model of human thermoregulation for a wide range of environmental conditions: the passive system. J Appl Physiol 1999, 87:1957-1972.

16. Bard P, ed: Medical physiology. St Louis: Mosby; 196I.

17. Duck FA: Physical properties of tissue. NewYork: Academic; 1990.

18. ESHO Task Group Committee: Treatment Planning and Modelling in Hyperthermia, Task Group Report of the European Society for Hyperthermic Oncology Rome: Tor Vergata; 1992.

19. Hardy J, Gagge A, Stolwijh J, eds: Physiological and behavioral temperature regulation. NewYork: Thomas; 1970:28I-30I.

20. ICRP: Report of the task group on reference man. In ICRP Publication 23 New York: ICRP; I 974.

21. National Institute for Longevity Sciences Longitudinal Study of Aging (NILS-LSA): Monograph-The Third Wave Chap. XI Anthropometry and Body Composition 2002.

22. Williams LR, Leggett RW: Reference values for resting blood flow to organs of man. Clinical Phys Physiol Meas 1989, I0:187-217.

23. Hirata A, Fujiwara O, Shiozawa T: Correlation between peak spatial-average SAR and temperature increase due to antennas attached to human trunk. IEEE Trans Biomed Eng 2006, 53:1658-1664.

24. IEEE C95-I: IEEE standard for safety levels with respect to human exposure to radio frequency electromagnetic fields, $3 \mathrm{kHz}$ to $300 \mathrm{GHz} .2005$. 